\title{
ATRIBUTOS FÍSICOS DE UM PLANOSSOLO HÁPLICO SOB SISTEMAS DE MANEJO COMPARADOS AOS DO CAMPO NATIVO ${ }^{(1)}$
}

\author{
Cláudia Liane Rodrigues de Lima ${ }^{(2)}$, Clenio Naito Pillon ${ }^{(3)}$, Luis Eduardo \\ Akiyoshi Sanches Suzuki ${ }^{(4)} \&$ Lucia Elena Coelho da Cruz ${ }^{(5)}$
}

\begin{abstract}
RESUMO
Sistemas de manejo podem influenciar as condições físico-hídricas do solo e a produtividade das culturas. O objetivo deste estudo foi avaliar os atributos físicos de um Planossolo Háplico sob diferentes sistemas de manejo e compará-los com os encontrados no campo nativo. A densidade, a condutividade hidráulica de solo saturado, a porosidade e a agregação foram avaliadas nas camadas de $0,000-0,025$; $0,025-0,075 ; 0,075-0,125 ; 0,125-0,175 ; 0,175-0,275 \mathrm{~m}$. O sistema plantio direto e o preparo convencional apresentaram condições físico-hídricas similares, enquanto o solo sob campo nativo proporcionou condições adequadas ao crescimento e desenvolvimento de plantas por apresentar maior condutividade hidráulica, macroporosidade, porosidade total e agregação, e menor densidade do solo. Sugerem-se valores críticos para as plantas, respectivamente de densidade, de condutividade hidráulica saturada, de diâmetro médio ponderado de agregados de $1,56 \mathrm{Mg} \mathrm{m}^{-3} ; 16,18 \mathrm{~mm} \mathrm{~h}^{-1}$ e $2,49 \mathrm{~mm}$.
\end{abstract}

Termos de indexação: densidade do solo, condutividade hidráulica de solo, porosidade do solo, agregação do solo.

\footnotetext{
(1) Recebido para publicação em janeiro de 2008 e aprovado em agosto de 2008.

(2) Professora, Departamento de Solos, Universidade Federal de Pelotas - UFPel. Campus Universitário s/n, Caixa Postal 354, CEP 96010-900 Pelotas (RS). E-mail: clrlima@yahoo.com.br

(3) Pesquisador, Embrapa Clima Temperado, BR 392, km 78, Caixa Postal 403, CEP 96001-970 Pelotas (RS). E-mail: pillon@cpact.embrapa.br

(4) Professor, Universidade Estadual do Rio Grande do Sul - UERGS. Rua Dr. José Bisognin 242, Bairro São Cristóvão, CEP 99700-000 Erechim (RS). E-mail: du_suzuki@hotmail.com

${ }^{(5)}$ Doutoranda, Universidade Federal de Pelotas. E-mail: luciaecruz@yahoo.com.br
} 


\title{
SUMMARY: PHYSICAL ATTRIBUTES OF AN ALBAQUALF UNDER MANAGEMENT SYSTEMS IN COMPARISON WITH THOSE UNDER NATIVE GRASSLAND
}

\begin{abstract}
The soil management may influence soil hydro-physical conditions and crop yield. The objective of this study was to determine soil physical properties of an Albaqualf under management systems and to compare them with those under a native pasture. The bulk density, saturated hydraulic conductivity, macroporosity, microporosity, total porosity and aggregation were evaluated in the soil layers $0-0.025 ; 0.025-0.075 ; 0.075-0.125 ; 0.125$ 0.175 ; and $0.175-0.275 \mathrm{~m}$. The hydro-physical soil conditions in no-till and conventional systems were similar. The conditions in the soil under native grassland were more adequate for crop development due to a higher hydraulic conductivity, macroporosity, total porosity and aggregation and lower bulk density. Critical values considered adequate for the crop development of $1.56 \mathrm{Mg} \mathrm{m}^{-3} ; 16.18 \mathrm{~mm} \mathrm{~h}^{-1}$ and $2.49 \mathrm{~mm}$, respectively, for bulk density, saturated hydraulic conductivity and mean weight diameter were suggested.
\end{abstract}

Index terms: bulk density, hydraulic conductivity, soil porosity, soil aggregation.

\section{INTRODUÇÃO}

Propriedades físicas têm sido utilizadas na avaliação da qualidade estrutural de solos. Terras baixas apresentam limitações naturais, que são intensificadas pelo cultivo e pelo tráfego de máquinas agrícolas (Lima et al., 2008; Louzada et al., 2008). Nessas áreas, sistemas de manejo inadequados têm alterado a densidade, a porosidade (Pedrotti et al., 2001; Lima et al., 2006) e a agregação do solo (Lima et al., 2003; Tavares Filho et al., 2005; Reichert et al., 2006).

A agregação é dependente de agentes cimentantes como polissacarídeos (agentes transitórios), raízes e hifas de fungos (agentes temporários) e cátions associados a compostos aromáticos recalcitrantes, adsorvidos a polímeros (agentes persistentes), os quais permanecem de forma diferenciada no solo (Tisdall \& Oades, 1982). Os sistemas de manejo, além de influenciar a agregação (Li et al., 2006; Keller et al., 2007), controlam a permanência desses agentes cimentantes. A permanência da cobertura vegetal sobre o solo pode diminuir a energia cinética imposta pelas gotas da chuva, diminuindo os processos de erosão e a perda de nutrientes e de água (Dedecek, 1989). A estabilidade estrutural tem importância no que se refere à umidade e à dinâmica de nutrientes do solo e à produtividade agrícola (Carter, 2002), mas não representa uma propriedade única na avaliação da degradação do solo (Boyx-Faios et al., 2001). A condutividade hidráulica tem sido útil na diferenciação dos efeitos de sistemas de preparo e na movimentação de água no perfil (Assis \& Lanças, 2005). Em um Planossolo, Lima et al. (2002) concluíram que o sistema natural apresentou condutividade hidráulica mais de $100 \%$ superior ao sistema de manejo convencional.

Estudos envolvendo solos de terras baixas do Rio Grande do Sul justificam-se pelo crescente interesse na identificação e no desenvolvimento de sistemas de uso e de manejo que propiciem a manutenção ou o incremento da qualidade física e da produtividade agrícola.

Propriedades físicas críticas ao crescimento e desenvolvimento de plantas têm sido apontadas por Lima et al. (2007). No entanto, ainda não existem valores de propriedades físicas consideradas críticas e limitantes do crescimento de culturas e que já estejam adequadamente validados na avaliação da qualidade de solos de várzea. $\mathrm{O}$ objetivo deste estudo foi avaliar atributos físicos de um Planossolo Háplico sob diferentes sistemas de manejo e compará-los com os encontrados no campo nativo, em busca de alternativas que viabilizem melhor aproveitamento e conhecimento das áreas de terras baixas com vistas à manutenção ou ao incremento da qualidade dos solos.

\section{MATERIAL E MÉTODOS}

O estudo foi realizado na Estação Experimental de Terras Baixas da Embrapa Clima Temperado, Capão do Leão, RS, $\left(31^{\circ} 49^{\prime}\right.$ Sul; $52^{\circ} 27^{\prime}$ Oeste, altitude 14 m), em um Planossolo Háplico (Embrapa, 2006) de textura superficial franco $\left(370 \mathrm{~g} \mathrm{~kg}^{-1}\right.$ de silte, $460 \mathrm{~g} \mathrm{~kg}^{-1}$ de areia e $170 \mathrm{~g} \mathrm{~kg}^{-1}$ de argila). O clima da região, de acordo com a classificação climática de Wilhelm Köppen, é do tipo Cfa (C: clima temperado quente, com temperatura média do mês mais frio entre 3 e $18^{\circ} \mathrm{C}$; f: em nenhum mês a precipitação pluvial é inferior a $60 \mathrm{~mm}$; a: temperatura do mês mais quente é superior a $22^{\circ} \mathrm{C}$ ).

Este estudo insere-se em um conjunto de ações experimentais programadas e implementadas em 2003. Na implantação do experimento, o solo foi revolvido com grade aradora (preparo primário) e grade niveladora (preparo secundário). No primeiro ano, a cultura de arroz irrigado foi utilizada para uniformizar o solo, seguido de pousio invernal. 
O delineamento experimental utilizado foi o de blocos ao acaso com parcelas divididas (três repetições), composto por cinco sistemas de cultura e dois sistemas de preparo de solo (plantio direto, PD e preparo convencional, PC). Os sistemas de cultura estão dispostos em parcelas (34 x $34 \mathrm{~m})$ e os sistemas de preparo de solo em subparcelas de 17 x $34 \mathrm{~m}$. Os sistemas de cultura contemplam culturas de interesse comercial no verão alternativas para o arroz irrigado, e culturas de cobertura no inverno, sendo três sistemas em sucessão e dois sistemas em rotação no verão. $\mathrm{O}$ manejo de adubação adotado esteve sempre baseado na recomendação de adubação de manutenção para as culturas de verão, utilizando-se fontes minerais de uréia, superfosfato triplo e $\mathrm{KCl}$, e de cobertura com $\mathrm{N}$, somente para o milho e o sorgo. No sistema PC, o manejo do solo foi realizado com grade aradora seguida de grade niveladora. A semeadura das culturas de verão foi realizada com auxílio de máquina semeadora em linha, e as culturas de cobertura de inverno semeadas a lanço nos dois sistemas de preparo (PD e PC).

Para o estudo de propriedades físicas, foram escolhidos dois sistemas de preparo do solo (PD e PC) e o sistema de cultura que contempla azevém (Lollium multiflorum Lam) + cornichão (Lotus corniculatus), como culturas de cobertura no inverno e rotação soja (Glycine Max L.)/milho (Zea mays L.)/sorgo (Sorghum vulgare) no verão. Em junho/2006, após cinco cultivos, com uma adição total de 13,7 e $20,92 \mathrm{Mg} \mathrm{ha}^{-1}$ de matéria seca para os sistemas PD e PC consecutivamente, foram coletadas amostras de solo com estrutura preservada e não-preservada. Amostras indeformadas de solo foram retiradas utilizando-se cilindros metálicos de duas dimensões $(0,05$ e 0,05 m; e 0,05 e 0,025 m, de diâmetro e altura, respectivamente). A amostragem foi efetuada em cinco camadas de solo (0,000-0,025; 0,025-0,075; 0,075-0,125; 0,125-0,175; 0,175-0,275 m). Como referência, amostrou-se uma área sob campo nativo $(\mathrm{CN})$, adjacente ao experimento, cujo tipo de solo, relevo e demais características são consideradas representativas da condição original da área experimental, anterior a seu uso agrícola.

Em laboratório, foram determinadas a densidade $\left(\mathrm{D}_{\mathrm{S}}\right)$ (Blake \& Hartge, 1986), a macroporosidade $\left(\mathrm{M}_{\mathrm{A}}\right)$, a microporosidade $\left(\mathrm{M}_{\mathrm{I}}\right)$ e a porosidade total $\left(\mathrm{P}_{\mathrm{T}}\right)$ do solo (Embrapa, 1997). A condutividade hidráulica de solo saturado $\left(\mathrm{K} \theta_{\mathrm{S}}\right)$ foi quantificada com o auxílio de um permeâmetro de carga constante (Libardi, 2005) em triplicata de laboratório.

A distribuição de agregados estáveis em água, em diferentes classes de tamanho (AEA, \%), e o diâmetro médio ponderado dos agregados (DMPA, mm) foi feita conforme o método descrito em Kemper \& Rosenau (1986) e Palmeira et al. (1999), que utiliza o aparelho de oscilação vertical Yoder (1936). O cálculo de agregados estáveis em água nas diferentes classes de tamanho $(4,76 ; 2,00 ; 1,00 ; 0,50 ; 0,25$ e menor que $0,25 \mathrm{~mm}$ ) e do diâmetro médio ponderado foi realizado utilizando, respectivamente, as equações 1 e 2 :

$$
\begin{array}{r}
A E A=\left[\frac{M A E A i-m i}{\sum_{i=1}^{n}(M A E A i-m i)}\right] \times 100 \\
D M P A=\left[\frac{\sum_{i=1}^{n} D M i \times(M A E A i-m i)}{\sum_{i=1}^{n}(M A E A i-m i)}\right]
\end{array}
$$

sendo: $M A E A i=$ massa de agregados $(g)+$ material inerte na classe $\mathrm{i}(\mathrm{g}) ; \mathrm{mi}=$ massa de material inerte na classe i (g); $D M I=$ diâmetro médio da classe i $(\mathrm{mm})$. Para separação de macro e de microagregados, adotouse o critério de Tisdall \& Oades (1982), que consideram o diâmetro de $0,25 \mathrm{~mm}$ como limite entre as classes.

Para avaliar os resultados obtidos, foram efetuados a análise de variância, o teste de comparação de médias, que considera a diferença mínima significativa, e a regressão linear simples a $5 \%$.

\section{RESULTADOS E DISCUSSÃO}

Houve significativa interação entre sistemas de manejo e camadas para a densidade, macroporosidade, microporosidade e porosidade total do solo (Quadro 1). Efeito significativo do manejo e da profundidade sobre a densidade e a porosidade do solo também foi indicado por Yavuzcan (2000).

O CN, com exceção da camada de 0,025 a $0,075 \mathrm{~m}$, apresentou o menor valor de Ds. Valores similares de Ds foram obtidos no sistema PD e no PC em todas as camadas de solo, com exceção de 0,125 a 0,175 m (Quadro 1). O incremento de Ds na camada de 0,125 a $0,175 \mathrm{~m}$ no sistema PD corrobora com conclusões de Pedrotti et al. (2001) sobre um Planossolo. Este fato deve-se ao acúmulo das pressões exercidas no trânsito de máquinas e à não-mobilização do solo com grade aradora e niveladora, efetuados anualmente antes da implantação das culturas de verão.

$\mathrm{A} \mathrm{M}_{\mathrm{A}}$ foi maior no $\mathrm{CN}$ em todas as camadas de solo, apresentando similaridade ao PD somente na camada de 0,025 a 0,075 m (Quadro 1). Possivelmente, os efeitos positivos do mínimo revolvimento de solo sobre a porosidade no sistema PD são significativos em anos subseqüentes à condução do experimento. Em comparação a outros sistemas de manejo, Lima et al. (2006) apresentaram maiores valores de porosidade total até a profundidade de $0,20 \mathrm{~m}$ em um Planossolo sob PD.

$\mathrm{O}$ sistema $\mathrm{PD}$ e o $\mathrm{PC}$ apresentaram resultados similares de $\mathrm{P}_{\mathrm{T}}$ nas camadas de 0,025 a 0,$075 ; 0,075$ a 0,$125 ; 0,175$ a $0,275 \mathrm{~m}$ (Quadro 1$)$. Os valores médios de $\mathrm{K} \theta$ s foram influenciados pelos sistemas de manejo $(p<0,0001)$, não havendo diferença entre as camadas avaliadas ( $<<0,5358)$. Resultados similares de KOs foram observados nos sistemas de PD e PC, apresentando-se superiores no CN (Quadro 1). A 
Quadro 1. Densidade $\left(D_{S}\right)$, macro $\left(M_{A}\right)$ e microporosidade $\left(M_{I}\right)$, porosidade total $\left(P_{T}\right)$ e condutividade hidráulica (KӨs) de um Planossolo Háplico sob sistemas de manejo

\begin{tabular}{|c|c|c|c|c|c|}
\hline \multirow{2}{*}{ Camada } & \multicolumn{4}{|c|}{ Sistema de manejo ${ }^{(1)}$} & \multirow{2}{*}{ Média } \\
\hline & PD & PC & & $\mathrm{CN}$ & \\
\hline $\mathrm{m}$ & \multicolumn{4}{|c|}{$\mathrm{D}_{\mathrm{S}}, \mathrm{Mg} \mathrm{cm}^{-3}$} & \\
\hline $0,000-0,025$ & $1,62 \mathrm{aB}$ & $1,61 \mathrm{aBC}$ & & $1,29 \mathrm{bB}$ & 1,51 \\
\hline $0,025-0,075$ & $1,55 \mathrm{aB}$ & $1,56 \mathrm{aC}$ & & $1,48 \mathrm{aA}$ & 1,53 \\
\hline $0,075-0,125$ & $1,66 \mathrm{aB}$ & $1,70 \mathrm{aAB}$ & & $1,57 \mathrm{bA}$ & 1,64 \\
\hline $0,125-0,175$ & $1,81 \mathrm{aA}$ & $1,69 \mathrm{bAB}$ & & $1,54 \mathrm{cA}$ & 1,68 \\
\hline $0,175-0,275$ & $1,82 \mathrm{aA}$ & $1,77 \mathrm{aA}$ & & $1,48 \mathrm{bA}$ & 1,69 \\
\hline Média & 1,69 & 1,67 & \multirow{6}{*}{$\mathrm{M}_{\mathrm{A}}, \mathrm{m}^{3} \mathrm{~m}^{-3}$} & 1,47 & \\
\hline $0,000-0,025$ & $0,16 \mathrm{bA}$ & $0,10 \mathrm{cA}$ & & $0,24 \mathrm{aA}$ & 0,17 \\
\hline $0,025-0,075$ & $0,08 \mathrm{abB}$ & $0,05 \mathrm{bB}$ & & $0,10 \mathrm{aB}$ & 0,07 \\
\hline $0,075-0,125$ & $0,06 \mathrm{bBC}$ & $0,04 \mathrm{bB}$ & & $0,10 \mathrm{aB}$ & 0,07 \\
\hline $0,125-0,175$ & $0,03 \mathrm{bC}$ & $0,04 \mathrm{bB}$ & & $0,11 \mathrm{aB}$ & 0,06 \\
\hline $0,175-0,275$ & $0,02 \mathrm{bC}$ & $0,03 \mathrm{bB}$ & & $0,12 \mathrm{aB}$ & 0,06 \\
\hline Média & 0,07 & 0,05 & \multirow{6}{*}{$\mathrm{M}_{\mathrm{I}}, \mathrm{m}^{3} \mathrm{~m}^{-3}$} & 0,13 & \\
\hline $0,000-0,025$ & $0,33 \mathrm{bA}$ & $0,32 \mathrm{bA}$ & & $0,44 \mathrm{aA}$ & 0,36 \\
\hline $0,025-0,075$ & $0,29 \mathrm{bB}$ & $0,32 \mathrm{aAB}$ & & $0,33 \mathrm{aB}$ & 0,31 \\
\hline $0,075-0,125$ & $0,29 \mathrm{aB}$ & $0,30 \mathrm{aBC}$ & & $0,29 \mathrm{aC}$ & 0,29 \\
\hline $0,125-0,175$ & $0,28 \mathrm{bB}$ & $0,31 \mathrm{aABC}$ & & $0,29 \mathrm{abC}$ & 0,29 \\
\hline $0,175-0,275$ & $0,29 \mathrm{aB}$ & $0,30 \mathrm{aC}$ & & $0,29 \mathrm{aC}$ & 0,29 \\
\hline Média & 0,30 & 0,31 & \multirow{7}{*}{$\mathrm{P}_{\mathrm{T}}, \mathrm{m}^{3} \mathrm{~m}^{-3}$} & 0,33 & \\
\hline $0,000-0,025$ & $0,49 \mathrm{bA}$ & $0,42 \mathrm{cA}$ & & $0,67 \mathrm{aA}$ & 0,53 \\
\hline $0,025-0,075$ & $0,37 \mathrm{bB}$ & $0,37 \mathrm{bB}$ & & $0,42 \mathrm{aB}$ & 0,39 \\
\hline $0,075-0,125$ & $0,35 \mathrm{bBC}$ & $0,34 \mathrm{bC}$ & & $0,39 \mathrm{aB}$ & 0,36 \\
\hline $0,125-0,175$ & $0,32 \mathrm{cC}$ & $0,35 \mathrm{bBC}$ & & $0,41 \mathrm{aB}$ & 0,36 \\
\hline $0,175-0,275$ & $0,32 \mathrm{bC}$ & $0,33 \mathrm{bC}$ & & $0,41 \mathrm{aB}$ & 0,35 \\
\hline Média & 0,37 & 0,36 & & 0,46 & \\
\hline $0,000-0,025$ & 2,33 & 2,56 & \multirow[t]{5}{*}{$\mathrm{K} \theta \mathrm{s}, \mathrm{mm} \mathrm{h}^{-1}$} & 24,34 & $9,74 \mathrm{~A}$ \\
\hline $0,025-0,075$ & 3,98 & 4,59 & & 21,44 & $10,01 \mathrm{~A}$ \\
\hline $0,075-0,125$ & 6,86 & 0,69 & & 7,27 & $4,94 \mathrm{~A}$ \\
\hline $0,125-0,175$ & 2,33 & 1,55 & & 18,28 & $7,39 \mathrm{~A}$ \\
\hline $0,175-0,275$ & 0,93 & 0,00 & & 20,68 & $7,20 \mathrm{~A}$ \\
\hline Média & $3,29 \mathrm{~b}$ & $1,88 \mathrm{~b}$ & & $18,40 \mathrm{a}$ & \\
\hline
\end{tabular}

(1) PD: plantio direto, PC: preparo convencional, CN: campo nativo. Médias seguidas pela mesma letra minúscula na linha e maiúsculas na coluna não diferem entre si pelo teste que considera a diferença mínima significativa a $\mathrm{p}<0,05$.

maior quantidade de raízes e de matéria orgânica, e a menor compactação do solo (menor densidade, maior macroporosidade e porosidade total) favoreceram aumento de $\mathrm{K} \theta$ s no CN.

Em comparação aos sistemas PD e PC, o maior DMPA foi observado no CN (Quadro 2). Valores maiores desta propriedade apresentaram-se principalmente nas camadas de 0,075 a 0,125 e de 0,125 a $0,175 \mathrm{~m}$. O maior DMPA no CN refletiu sobre a maior e a menor quantidade de macroagregados e de microagregados do solo, respectivamente (Quadro 2). A macroagregação dos sistemas seguiu, respectivamente, a tendência: $\mathrm{CN}>\mathrm{PD}>\mathrm{PC}$. Em comparação ao PD e ao CN, o PC causa a quebra de agregados de maior tamanho pelo intensivo revolvimento do solo (Tisdall \& Oades, 1982; Jiao et al., 2006).

Até a profundidade de $0,175 \mathrm{~m}$, observou-se uma percentagem similar de agregados retidos nas peneiras com diâmetro de 8,00 a 4,76; 4,76 a 2,00; 2,00 a 1,00 e $<0,25 \mathrm{~mm}$ (Figura 1a,b,c,d). A menor concentração de agregados em classes de menor tamanho e, por sua vez maior quantidade de agregados de maior tamanho no CN devem-se, possivelmente, à maior quantidade de matéria orgânica e de raízes, as quais contribuem na agregação pela aproximação das partículas do solo (Carter, 2002; Six et al., 2002). Watts et al. (1997) indicam que as pastagens normalmente incrementam o teor de matéria orgânica e a estabilidade de agregados do solo.

A partir da quantificação das propriedades físicas do solo, foram estabelecidas equações lineares de regressão. Verificou-se pelo coeficiente de determinação $\left(\mathrm{R}^{2}\right)$ que a variação da $\mathrm{M}_{\mathrm{A}}$, da $\mathrm{K} \theta$ s e do DMPA foram dependentes da Ds em, respectivamente, 63, 37 e $20 \%$. A $M_{A}$, a $K \theta$ s e o DMPA estiveram inversamente associados à Ds, indicado pelos valores negativos dos coeficientes angulares de ajuste dos modelos (Figura 2, Quadro 3). Esses resultados sugerem uma estimativa adequada de valores críticos de propriedaes físicohídricas no crescimento e desenvolvimento das plantas a partir de valores de aeração e densidade do solo.

Considerando-se que a $\mathrm{M}_{\mathrm{A}}$ foi sensivelmente influenciada pela Ds $(\mathrm{F}=150,52 ; \mathrm{CV}=43,62 \%) \mathrm{e}$ uma $\mathrm{M}_{\mathrm{A}}$ de $0,10 \mathrm{~m}^{3} \mathrm{~m}^{-3}$, valor sugerido por Grable \& Siemer (1968) como crítico ao crescimento radicular, em função da limitada difusão de $\mathrm{O}_{2}$ no solo para as plantas, estimou-se uma densidade crítica de aproximadamente $1,56 \mathrm{Mg} \mathrm{m}^{-3}$ para a camada de 
Quadro 2. Diâmetro médio ponderado de agregados (DMPA), macro e microagregados de um Planossolo Háplico sob sistemas de manejo

\begin{tabular}{|c|c|c|c|c|}
\hline \multirow{2}{*}{ Camada } & \multicolumn{3}{|c|}{ Sistema de manejo ${ }^{(1)}$} & \multirow[t]{2}{*}{ Média } \\
\hline & PD & PC & $\mathrm{CN}$ & \\
\hline $\mathrm{m}$ & \multicolumn{4}{|c|}{ DMPA, $\mathrm{mm}$} \\
\hline $0,000-0,025$ & 1,08 & 0,86 & 3,08 & $1,67 \mathrm{~B}$ \\
\hline $0,025-0,075$ & 1,25 & 0,97 & 2,81 & $1,68 \mathrm{~B}$ \\
\hline $0,075-0,125$ & 1,65 & 3,22 & 2,93 & $2,60 \mathrm{~A}$ \\
\hline $0,125-0,175$ & 1,46 & 1,01 & 3,74 & $2,07 \mathrm{AB}$ \\
\hline $0,175-0,275$ & 0,83 & 0,45 & 2,64 & $1,31 \mathrm{~B}$ \\
\hline \multirow[t]{2}{*}{ Média } & $1,25 \mathrm{~b}$ & $1,30 \mathrm{~b}$ & $3,04 \mathrm{a}$ & \\
\hline & \multicolumn{4}{|c|}{ Macroagregados, \% } \\
\hline $0,000-0,025$ & 39,58 & 36,37 & 77,25 & $51,07 \mathrm{~B}$ \\
\hline $0,025-0,075$ & 44,09 & 42,02 & 75,37 & $53,83 \mathrm{~B}$ \\
\hline $0,075-0,125$ & 53,09 & 52,25 & 77,28 & $60,87 \mathrm{~A}$ \\
\hline $0,125-0,175$ & 53,79 & 51,60 & 82,13 & $62,51 \mathrm{~A}$ \\
\hline $0,175-0,275$ & 48,66 & 36,94 & 70,77 & $52,12 \mathrm{~B}$ \\
\hline \multirow[t]{2}{*}{ Média } & $47,84 \mathrm{~b}$ & $43,84 \mathrm{c}$ & $76,56 \mathrm{a}$ & \\
\hline & \multicolumn{4}{|c|}{ Microagregados, \% } \\
\hline $0,000-0,025$ & 60,42 & 63,63 & 22,75 & $48,93 \mathrm{~A}$ \\
\hline $0,025-0,075$ & 55,90 & 57,98 & 24,63 & $46,17 \mathrm{~A}$ \\
\hline $0,075-0,125$ & 46,91 & 47,75 & 22,72 & $39,13 \mathrm{~B}$ \\
\hline $0,125-0,175$ & 46,21 & 48,40 & 17,87 & $37,49 \mathrm{~B}$ \\
\hline $0,175-0,275$ & 51,34 & 63,06 & 29,23 & $47,88 \mathrm{~A}$ \\
\hline Média & $52,16 \mathrm{~b}$ & $56,16 \mathrm{a}$ & $23,44 \mathrm{c}$ & \\
\hline
\end{tabular}

(1) PD: plantio direto, PC: preparo convencional, CN: campo nativo. Médias seguidas pela mesma letra minúscula na linha e maiúsculas na coluna não diferem entre si pelo teste que considera a diferença mínima significativa a $\mathrm{p}<0,05$.
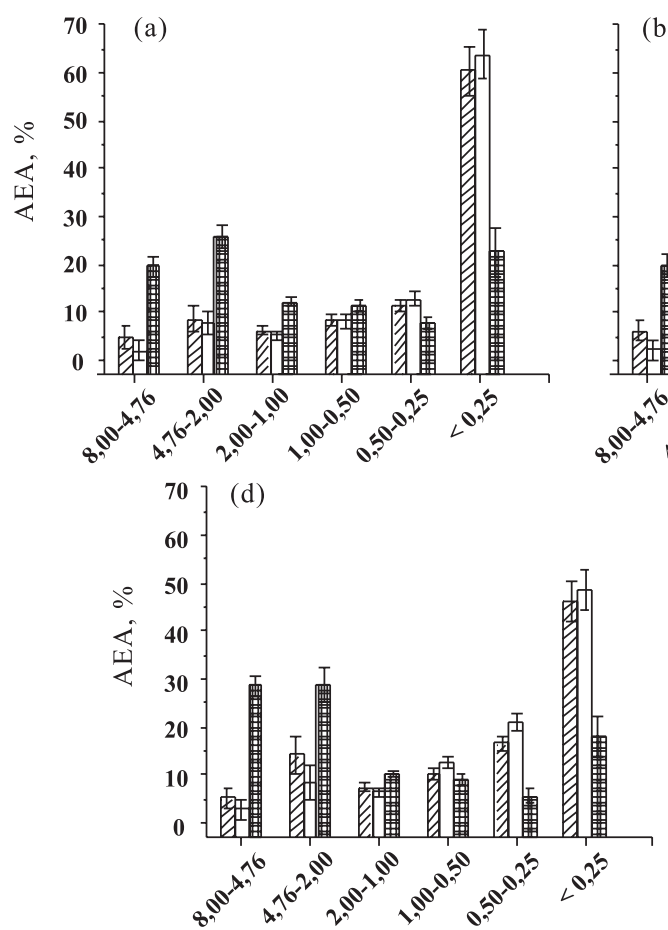

(b)
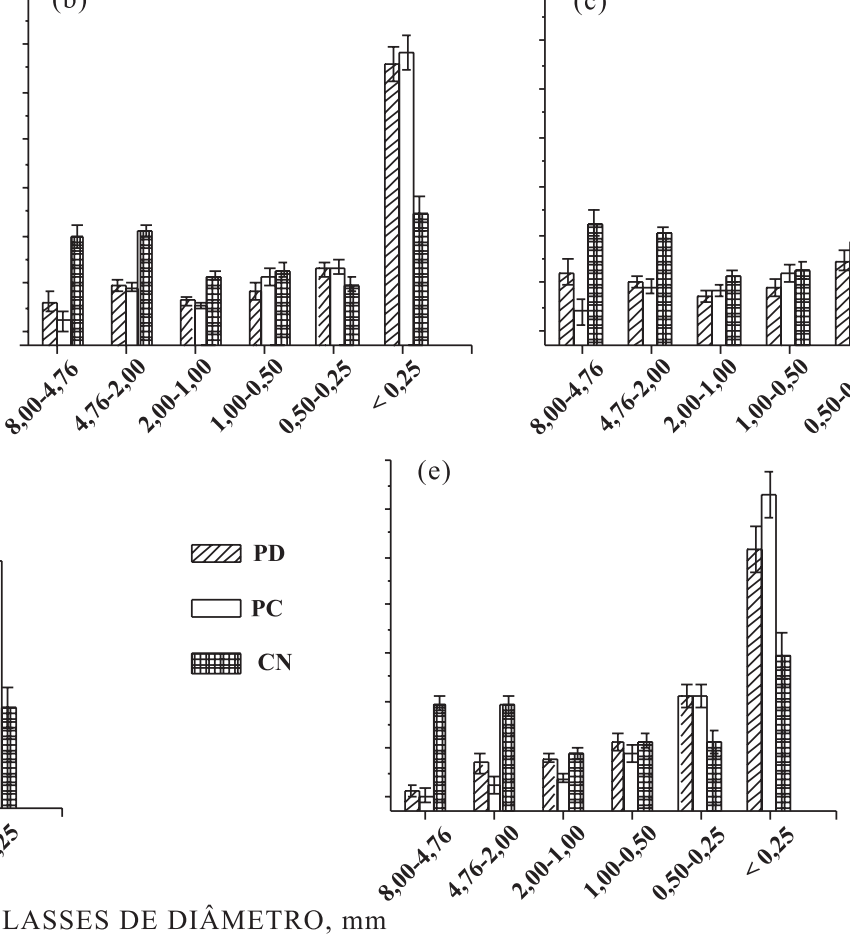

CLASSES DE DIÂMETRO, mm (c)

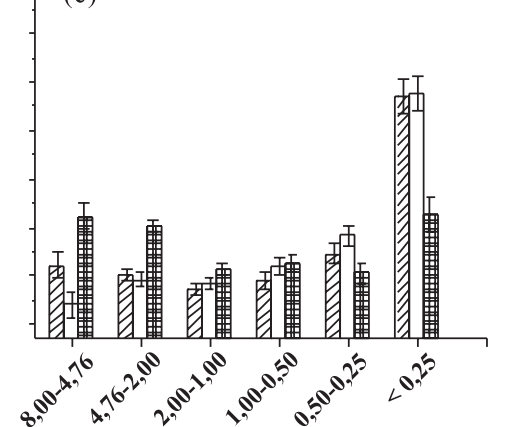

Figura 1. Agregados do solo estáveis em água (AEA) nas diferentes classes de diâmetro, nas camadas: (a) 0,000-0,025; (b) 0,025 a 0,075; (c) 0,075-0,125; (d) 0,125 a 0,175 e (e) 0,175 a 0,275 m de um Planossolo Háplico sob sistemas de manejo. PD: plantio direto, PC: preparo convencional, $\mathrm{CN}$ : campo nativo. Barras verticais indicam a diferença mínima significativa a $5 \%$ em cada classe de agregados entre os sistemas de manejo do solo.

0,000 a $0,275 \mathrm{~m}$ (Figura 2). Valores restritivos para diferentes tipos de solos e teores de argila têm sido apresentados por Lima et al. (2007) e Suzuki et al. (2006). Silva et al. (2006) concluíram que a densidade 


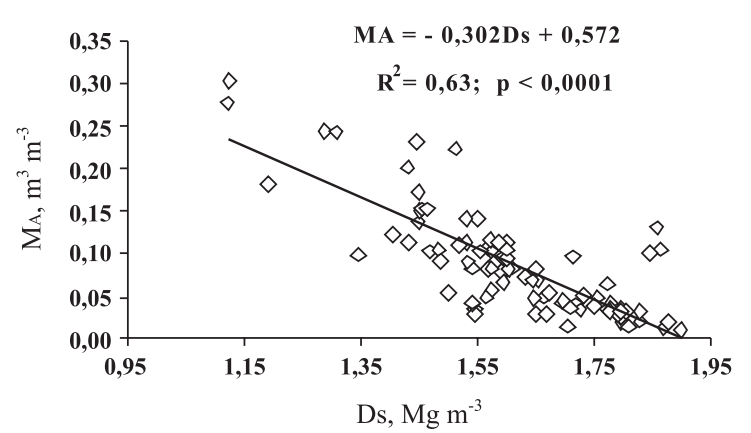

Figura 2. Relação obtida entre macroporosidade $\left(M_{A}\right)$ e densidade $\left(\mathrm{D}_{\mathrm{S}}\right)$ de um Planossolo Háplico.

Quadro 3. Parâmetros dos modelos: (i) $D s=$ $a+b \times K \theta s\left(\mathrm{p}<0,0001 ; \mathrm{F}=52,40 ; \mathrm{CV}=8,08 \% ; \mathrm{R}^{2}=\right.$ 0,37) e (ii) $D s=a+b \times D M P A(\mathrm{p}<0,0001 ; \mathrm{F}=$ 22,89; $\left.\mathrm{CV}=9,12 \% ; \mathrm{R}^{2}=0,20\right)$ de um Planossolo Háplico

Parâmetro Valor estimado Erro-padrão Valor t $\mathrm{Pr}>\mathrm{t}$

\begin{tabular}{cccrc}
\hline & \multicolumn{5}{c}{ i } & \\
$\mathrm{a}$ & 1,6690 & 0,01592 & 104,85 & $<0,0001$ \\
$\mathrm{~b}$ & $-0,00677$ & 0,0009 & $-7,24$ & $<0,0001$ \\
& & $\mathrm{ii}$ & & \\
$\mathrm{a}$ & 1,7272 & 0,0289 & 59,61 & $<0,0001$ \\
$\mathrm{~b}$ & $-0,0683$ & 0,0142 & $-4,78$ & $<0,0001$ \\
\hline
\end{tabular}

de $1,50 \mathrm{Mg} \mathrm{m}^{-3}$ comprometia o crescimento da parte aérea de soja, do algodão e do milho em um Latossolo.

A partir da $\mathrm{Ds}=1,56 \mathrm{Mg} \mathrm{m}^{-3}$ e dos modelos de regressão (Quadro 3), quantificaram-se os valores de K$\theta$ s e de DMPA considerados críticos ao crescimento radicular, obtendo-se $16,18 \mathrm{~mm} \mathrm{~h}^{-1}$ para a $\mathrm{K} \theta \mathrm{s}$ e 2,49 $\mathrm{mm}$ para o DMPA. Suzuki et al. (2007), considerando uma porosidade de aeração de $0,10 \mathrm{~m}^{3} \mathrm{~m}^{-3}$ em um Argissolo, encontraram um valor crítico de $\mathrm{K} \theta \mathrm{s}$ no crescimento de culturas semelhante $\left(17,38 \mathrm{~mm} \mathrm{~h}^{-1}\right)$ ao obtido neste estudo.

De forma geral, considerando-se os valores médios e críticos das propriedades físicas (Quadros 1, 2 e 3 e Figura 2), somente o solo sob CN apresenta condições mais adequadas ao crescimento de plantas. $\mathrm{O}$ estabelecimento de relações simples e quantitativas a partir da Ds apresenta vantagem por ser um processo rápido, de fácil mensuração e de baixo custo de avaliação. No entanto, estudos adicionais devem ser implementados em solos de terras baixas para melhor entendimento e validação dos valores críticos de propriedades físicas quanto ao suprimento adequado de $\mathrm{O}_{2}$ para organismos animais e vegetais. $\mathrm{O}$ monitoramento da qualidade estrutural em ambiente de terras baixas constitui etapa importante na definição e nos ajustes de práticas conservacionistas de manejo que garantam a manutenção e a melhoria da qualidade do solo.

\section{CONCLUSÕES}

O sistema plantio direto e o preparo convencional apresentaram condições físico-hídricas similares, enquanto o solo, sob campo nativo, proporcionou condições mais adequadas ao crescimento de plantas por apresentar maior condutividade hidráulica, macroporosidade, porosidade total e agregação e menor densidade do solo. Os valores críticos ao crescimento de plantas, respectivamente de densidade, de condutividade hidráulica saturada, e de diâmetro médio ponderado de agregados foram $1,56 \mathrm{Mg} \mathrm{m}^{-3}$; $16,18 \mathrm{~mm} \mathrm{~h}^{-1}$ e $2,49 \mathrm{~mm}$.

\section{LITERATURA CITADA}

ASSIS, R.L. \& LANÇAS, K.P. Avaliação dos atributos físicos de um Nitossolo Vermelho distroférrico sob sistema plantio direto, preparo convencional e mata nativa. R. Bras. Ci. Solo, 29:512-522, 2005.

BLAKE, G.R. \& HARTGE, K.H. Bulk density. In: KLUTE, A., ed. Methods of soil analysis: Physical and mineralogical methods. 2.ed. Madison, American Society of Agronomy, Soil Science Society of America, 1986. p.363-375.

BOYX-FAYOS, C.; CALVO-CASES, A.; IMENSON, A.C. \& SORIANO-SOTO, M.D. Influence of soil properties on the aggregation of some Mediterranean soils and the use of aggregate size and stability as land degradation indicators. Catena, 44:47-67, 2001.

CARTER, M.R. Soil quality for sustainable land management: Organic matter and aggregation interactions that maintain soil functions. Agron. J., 94:38-47, 2002.

DEDECEK, R.A. Coberturas permanentes do solo na erosão sob condições de cerrado. Pesq. Agropec. Bras., 24:483488, 1989.

EMPRESA BRASILEIRA DE PESQUISA DE PESQUISA AGROPECUÁRIA - EMBRAPA. Centro Nacional de Pesquisa de Solos. Manual de métodos de análise de solo. 2.ed. Rio de Janeiro, 1997. 212p.

EMPRESA BRASILEIRA DE PESQUISA DE PESQUISA AGROPECUÁRIA - EMBRAPA. Centro Nacional de Pesquisa de Solos. Sistema Brasileiro de Classificação dos Solos. 2. ed. Rio de Janeiro: Embrapa Solos, 2006. 306p.

GRABLE, A.R. \& SIEMER, E.G. Effects of bulk density aggregate size and soil water suction on oxygen diffusion, redox potential and elongation of corn roots. Soil Sci. Soc. Am. J., 32:18-186, 1968.

JIAO, Y.; WHALEN, J.K. \& HENDERSHOT, W.H. No tillage and manure applications increase aggregation and improve nutrient retention in a sandy-loam soil. Geoderma, 134:24-33, 2006.

KELLER, T.; ARVIDSSON, J. \& DEXTER, A.R. Soil structures produced by tillage as affected by soil water content and the physical quality of soil. Soil Till. Res., 92:45-52, 2007. 
KEMPER, W.D. \& ROSENAU, R.C. Aggregate stability and size distribution. In: KLUTE, A., ed.. Methods of soil analysis. 2.ed. Madison, Wisconsin, American Society of Agronomy, Soil Science Society of America, 1986. p.425441.

LI, X.G.; LI, F.M.; RENGEL, Z.; SINGH, B. \& WANG, Z.F. Cultivation effects on temporal changes of organic carbon and aggregate stability in desert soils of Hexi Corridor region in China. Soil Till. Res., 91:22-29, 2006.

LIBARDI, P.L. Dinâmica da água no solo. São Paulo, Universidade de São Paulo, 2005. 335p.

LIMA, A.C.R.; HOOGMOED, W. \& BRUSSARD, L. Soil quality assessment in rice production systems: Establishing a minimum data set. J. Environ. Qual., 37:623-630, 2008.

LIMA, C.L.R.; PAULETTO, E.; GOMES, A.S.; HARTWIG, M.P. \& PASSIANOTO, C.C. Compactação de um Planossolo em função de sistemas de manejo. R. Bras. Agroci., 12:179182,2006

LIMA, C.L.R.; PAULETTO, E.A.; GOMES, A.S. \& SILVA, J.B. Estabilidade de agregados de um Planossolo sob diferentes sistemas de manejo. R. Bras. Ci. Solo, 27:199-205, 2003.

LIMA, A.C.R.; PAULLETO, E.A.; LIBARDI, P.L.; SILVA, A.G. \& PINTO, L.F.S. Hydraulic characterization of a lowland soil under long-term management systems. Adv. Geoecol., 35:247-257, 2002 .

LIMA, C.L.R.; REINERT, D.; REICHERT, J.M.; SUZUKI, L.E.A.S. \& HILBIG, V.S. Densidade critica ao crescimento de plantas considerando água disponível e resistência à penetração de um Argissolo Vermelho distrófico arênico. Ci. Rural, 37:1166-1169, 2007.

LOUZADA, J.A.; CAICEDO, N. \& HELFER, F. Condições de drenagem relacionadas ao trânsito de máquinas em solo de várzea (RS-Brasil). Rev. Bras. Eng. Agric. Amb., 12:98$106,2008$.

PALMEIRA, P.R.T.; PAULETTO, E.A.; TEIXEIRA C.F.A.; GOMES A.S. \& SILVA, J.B. Agregação de um Planossolo submetido a diferentes sistemas de cultivo. R. Bras. Ci. Solo, 23:189-195, 1999.

PEDROTTI, A.; PAULETTO, E.A.; GOMES, A.S.; TURATTI, A.L. \& CRESTANA, S. Sistemas de cultivo de arroz irrigado e a compactação de um Planossolo. Pesq. Agropec. Bras., 36:709-715, 2001.
REICHERT, J.M.; LIMA, C.L.R.; DALMOLIN, R.S.D.; REINERT, D.J.; GONÇALVES, C. \& NUNES, M. Agregação de um Planossolo sistematizado há um ano e sob cultivo de arroz irrigado. Ci. Rural, 36:837-844, 2006.

SILVA, G.J.; MAIA, J.C.S. \& BIANCHINI, A. Crescimento da parte aérea de plantas cultivadas em vaso, submetidas à irrigação subsuperficial e a diferentes graus de compactação de um Latossolo Vermelho-Escuro distrófico. R. Bras. Ci. Solo, 30:31-40, 2006.

SIX, J.; FELLER, C.; DENEF, K.; OGLE, S.M.; SA M.J.C. \& ALBRECHT, A. Soil organic matter, biota, and aggregation in temperate and tropical soils - Effect of no tillage. Agronomie, 22:755-775, 2002.

SUZUKI, L.E.A.S.; REICHERT, J.M.; REINERT, D. \& LIMA, C.L.R. Grau de compactação, propriedades físicas e rendimento de culturas em Latossolo e Argissolo. Pesq. Agropec. Bras., 42:1159-1167, 2007.

SUZUKI, L.E.A.S.; REINERT, D.; REICHERT, J.M. \& LIMA, C.L.R. Densidade restritiva ao crescimento radicular em função da argila. In: REUNIÃO BRASILEIRA DE MANEJO E CONSERVACC̃̃O DO SOLO E DA ÁGUA, NOVOS DESAFIOS DO CARBONO DO MANEJO CONSERVACIONISTA, 16., Aracaju, 2006. Anais. Aracaju, 2006. CD-ROM.

TAVARES FILHO, J.; GRIMALDI, M. \& TESSIER, D. Compressibilidade de agregados de um Latossolo Amarelo da Amazônia em resposta ao potencial da água do solo. R. Bras. Ci. Solo, 29:489-495, 2005.

TISDALL, J.M. \& OADES, J.M. Organic matter and water stable aggregates in soils. J. Soil Sci., 33:141-163, 1982.

YAVUZCAN, H.G. Wheel traffic impact on soil condition as influenced by tillage in Central Anatolia. Soil Till. Res., 54:129-138, 2000.

YODER, R.E. A direct method of aggregate analysis of soils and a study of the physical nature of erosion losses. J. Am. Soc. Agron., 28:337-351, 1936.

WATTS, C.W. \& DEXTER, A.R. The influence of organic matter in reducing the destabilization of soil by simulated tillage. Soil Till. Res., 42:253-275, 1997. 